\title{
Modeling and analysis of engaging process of automated mechanical transmissions
}

\author{
Hongxu Chen $^{1}$ (D) - Guangyu $\operatorname{Tian}^{1}$
}

Received: 4 May 2015 / Accepted: 30 November 2015 / Published online: 23 December 2015

(C) The Author(s) 2015. This article is published with open access at Springerlink.com

\begin{abstract}
In automated mechanical transmissions, engaging sleeve with clutch gear causes the switches of mechanical coupling modes of engaging-related parts, and impacts may happen to make the part speeds have discrete transitions during the engaging. The two characters make the engaging process exhibit both continuity and discreteness. The aim of the paper is to develop a high-fidelity model to capture the trajectories of the parts in the transmission. We treat the engaging as a two-phase process—-sleeve first interacting with synchro ring and then with clutch gear. The part movements under a certain constraint are governed by multibody dynamics, and the speed jumps caused by impacts are described using the Poisson coefficient of restitution. To couple the continuous evolutions and discrete transitions of the states, a hybrid automaton model is developed. This model allows for the characteristic analysis of the engaging process with multiple interaction cases between the engaging-related parts, and it is validated by bench tests. The effects of two key factors-the relative tooth position of sleeve and clutch gear and the shifting force-on engaging duration and impact are studied through numerical simulations.
\end{abstract}

Keywords Multibody dynamics · Hybrid system - Automated mechanical transmission · Shift gear · Impact

\section{Introduction}

Engaging sleeve with clutch gear is the key process of shifting gears in automated mechanical transmissions (AMT). It occupies more than $70 \%$ power off time of a gear shift in an AMT, and undesirable impacts may happen during an engaging $[2,13]$. As shown in Fig. 1a, for shifting gears, a shift mechanism pushes the sleeve to move axially disengaging from an

\footnotetext{
$凶$ H. Chen

herschel.chen@gmail.com

G. Tian

tian_gy@tsinghua.edu.cn

1 State Key Laboratory of Automotive Safety and Energy, Tsinghua University, 100084 Beijing, P.R. China
} 


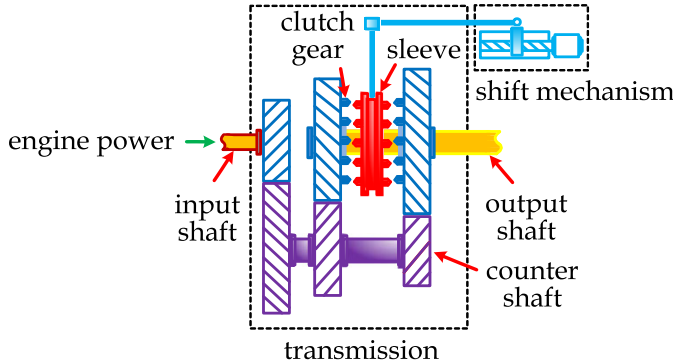

(a) Kinetic chain

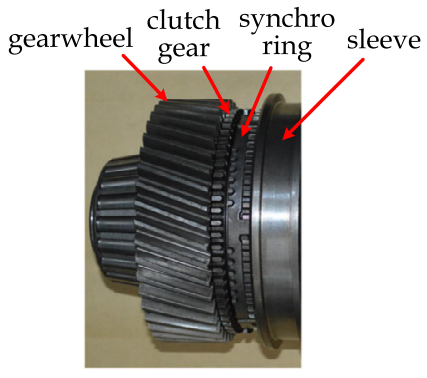

(b) Assembly

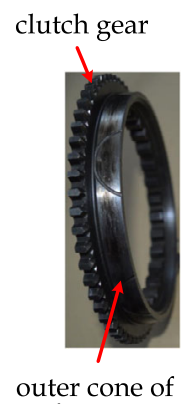

friction

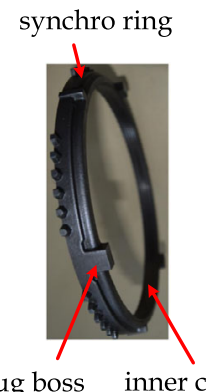

lug boss friction

splined hub output shaft

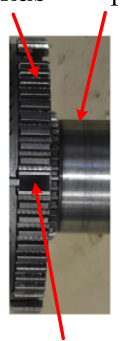

straight slot

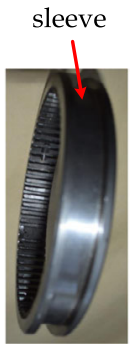

(c) Exploded view

Fig. 1 A synchro-ring-type synchronizer includes a sleeve, a synchro ring, a clutch gear, etc.

original clutch gear and then engaging with a desired clutch gear. If the sleeve arrives at the desired clutch gear at an improper rotational tooth position, it would lead to an impact, which makes the engaging delayed and jerky [1, 3, 6, 11]. Worse still, a big rotational speed difference between the sleeve and the clutch gear causes repeated impacts, which cause grating or scraping noises and damage their splined teeth $[9,15]$. In order to shorten the power off time and reduce the impact caused by shifting gears, careful modeling and analysis for the engaging process is needed.

In this paper, we take the synchro-ring-type synchronizer as an example (Fig. 1), and model its engaging process of sleeve and clutch gear. As shown in Fig. 1b, this type of synchronizer mainly includes a sleeve, a synchro ring and a clutch gear, and they are installed coaxially. As shown in Fig. 1c, the sleeve and the output shaft are connected mechanically at the rotational direction through a splined hub, and the sleeve could move axially along the splined hub. The clutch gear is fixed on the gearwheel, which meshes with the input shaft of transmission through the counter shaft (Fig. 1a). The synchro ring is installed between the sleeve and the clutch gear.

The engaging-related parts of a synchronizer interact with each other during an engaging. Before engaging, the sleeve is at neutral gear, where it is disengaged from an original clutch gear. For engaging sleeve with a desired clutch gear, a shift mechanism pushes the sleeve to move forward axially to first interact with the synchro ring and then with the clutch gear. When the inner cone of friction of the synchro ring contacts with the outer cone of friction of the clutch gear, a frictional torque arises to rotate the lug boss of the synchro ring relative to the splined hub (sleeve) if the sleeve and the clutch gear are asynchronous. 
This rotation makes the synchro ring teeth stand on the way of the sleeve teeth. When the sleeve teeth arrive at the tooth chamfers of the synchro ring, a bigger frictional torque arises to synchronize the sleeve and the clutch gear. Once they are synchronous exactly, the sleeve pushes the synchro ring to rotate an angle and goes through the synchro ring, and then the sleeve interacts with the clutch gear. Finally, when the sleeve teeth arrive at the tooth spaces of the clutch gear, the engaging completes, and the clutch gear is coupled with the output shaft through the sleeve, and as a result the engine power recovers to transmit to the output shaft with a new gear ratio. So, engaging sleeve with clutch gear causes the switches of mechanical coupling modes of the engaging-related parts.

Controlling the sleeve to engage with the clutch gear faces a dilemma. To shorten the power off time, the sleeve should move as quickly as possible, but if it moves too quickly, then the synchro ring has no enough time to rotate a half tooth thickness forward and fail to lock out the sleeve. This "lock-out" failure leads to a big rotational speed difference between the sleeve and the clutch gear when they interact with each other, and a high impact happens if their splined teeth are misaligned. This impact then makes the speeds of the sleeve and the clutch gear jump discretely. More specifically, the states of the engaging-related parts evolve continuously until the sleeve teeth collide with the clutch gear teeth, at which point their axial and rotational speeds have discrete transitions, after which their states continue to evolve continuously again.

The switches of mechanical coupling modes and the impacts between the engagingrelated parts make the engaging process exhibit both continuity and discreteness. For different mechanical coupling modes, the system dynamics cannot be modeled through a unified equation. Instead, we need multiple equation sets to formulate the system dynamics and switching conditions to switch the mechanical coupling mode. For each impact, a mathematical model is needed to capture the speed jumps of the engaging-related parts.

Previous works on the modeling and analysis of engaging process are based on phase division. Socin et Walters [14] divide the operation of the sleeve from neutral to full engagement into six phases and present the system dynamics of each phase. For higher accuracy, Lovas et al. $[6,7]$ divide the engaging process into eight phases and make clear that the shifting force has a significant influence on the impacts during an engaging by causing different relative tooth positions of the sleeve and the desired clutch gear. Statistically, Liu et al. [5] reduce the uncertain relative tooth positions to four cases and obtain the probabilities of the cases by lots of simulation experiments. A multibody dynamic model in [3] mimics the engaging process, and simulation results reveal that impact happens when the sleeve moves backwards. In order to model the impacts between the tooth chamfers of the sleeve and the clutch gear, Kim et al. [4] abstract the contact mechanism into a linear spring and obtain the spring stiffness by finite element method. These modeling and analysis works are based on empirical hypotheses to some extent, and the independent phase-by-phase analyses ignore many possible engaging cases, which cannot reveal the mechanism of impacts theoretically.

In this work, we present a methodology for modeling the engaging process from hybrid system theory and then analyze the engaging characteristics through numerical simulations. When the engaging-related parts of a synchronizer are under a certain constraint, we model their dynamics with the multibody dynamics theory. When an impact happens between them, we describe the impact process with the Poisson coefficient of restitution and then develop an impact model to capture their speed jumps as the impact process is approximately a rigid-body collision, duration of which is very short and the part deformations are extremely weak. In order to couple the state continuous evolutions and discrete transitions, we develop a hybrid automaton (HA) model to capture the trajectories of the engagingrelated parts, and this model is validated by bench tests. Based on the validated model, we 
carry out simulations in MATLAB with different initial states and inputs. Our results show that when the relative tooth position of sleeve and clutch gear is different, the sleeve and the clutch gear have five possible interaction ways, which result in different engaging duration and impact impulse. In addition, when the shifting force increases, the engaging duration has a downtrend, and the impact impulse has a rising trend, but the generating mechanism of the maximum impact switches when the shifting force is bigger than a threshold value.

We begin with a presentation of the engaging process in Sect. 2, where we illustrate two interaction phases. In Sect. 3, we build a high-fidelity HA model for the engaging process, and its detailed multibody dynamic equations are attached in Appendix B. Section 4 is concerned with the characteristic analysis of the engaging process. The paper closes with concluding remarks in Sect. 5.

\section{Presentation of engaging process}

As impacts happen to block the axial movement of the sleeve during its engaging with the clutch gear, a detailed model is needed to analyze the characteristics of the engaging process and reveal the impact mechanism. The configuration of the synchro-ring-type synchronizer is shown in Fig. 2. In order to illustrate part positions during engaging, only the splines of the sleeve and the clutch gear, and the splines and the lug boss of the synchro ring are shown (Fig. 2a). Note that the inner view of the sleeve is given in order to facilitate visualization. The configuration parameters of the transmission system are specified in Appendix A. Based on the interaction ways of the engaging-related parts, we treat the engaging of sleeve and desired clutch gear as a two-phases process-sleeve first interacting with synchro ring and then with clutch gear.

The force analyses of engaging-related parts are shown in Fig. 3. As shown in Fig. 3b, the shifting force $F_{s}$ acts on the sleeve such that the sleeve and the sliding block move forward axially together through the spring plunger. As shown in Fig. 3a, after the left end face of the sliding block contacts with the right end face of the synchro ring, the sliding block pushes the synchro ring to move axially towards the clutch gear. As the lug boss of the synchro ring stops at the upper slot wall initially (Fig. 2a), it has a same rotational speed with the

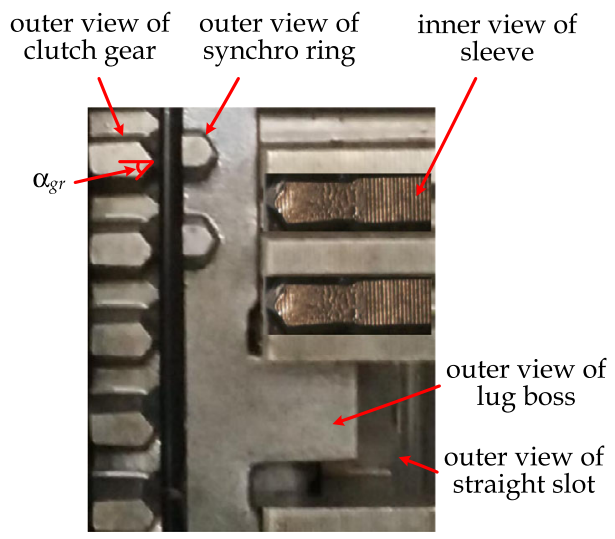

(a) Splines and lug boss

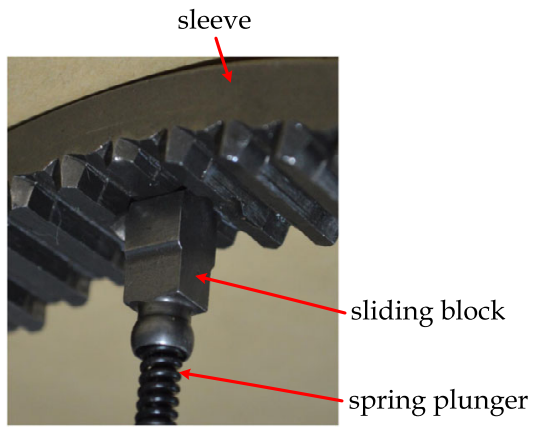

(b) Positioning device

Fig. 2 The configuration of a synchro-ring-type synchronizer 


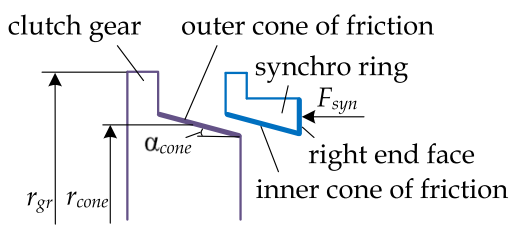

(a) Free-body diagrams of synchro ring and clutch gear

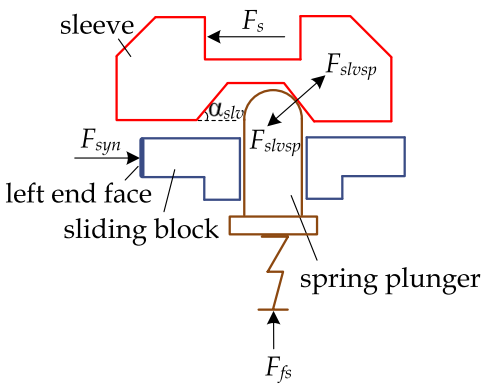

(b) Free-body diagrams of sleeve, spring plunger and sliding block

Fig. 3 Force analyses of engaging-related parts

sleeve, that is, $\omega_{s y n}=\omega_{s l v}$, where $\omega_{s y n}$ represents the rotational speed of the synchro ring, and $\omega_{s l v}$ represents the rotational speed of the sleeve. When the inner cone of friction of the synchro ring contacts with the outer cone of the clutch gear, the synchro ring stops its axial movement, but the axial pushing force $F_{s y n}$ still acts on the synchro ring so that the two cones of friction keep contacting and a relative rotation may happen. At the axial direction, the sleeve continues its movement until hitting upper or lower tooth chamfers of the synchro ring.

\subsection{Interaction of sleeve and synchro ring}

The interaction way of the sleeve and the synchro ring is determined by the relative rotational speed of the sleeve and the clutch gear $\Delta \omega_{s l v-g r}$ and the relative tooth position of the sleeve and the synchro ring $\Delta \theta_{s l v-s y n}$, where $\Delta \omega_{s l v-g r}=\omega_{s l v}-\omega_{g r}$ and $\Delta \theta_{s l v-s y n}=\theta_{s l v}-\theta_{s y n}$. Since the straight slot width equals the sum of a lug boss and a tooth thickness, the $\Delta \theta_{s l v-s y n}$ is within the domain of $[-\pi / 2 N,+\pi / 2 N]$, where $N$ is the splined tooth number of a sleeve. We analyze the interaction process of the sleeve and the synchro ring with five cases.

(1) If the relative rotational speed of sleeve and desired clutch gear is small enough, that is, $\left|\Delta \omega_{s l v-g r}\right| \leq \varepsilon_{\omega}\left(\varepsilon_{\omega} \rightarrow 0^{+}\right)$, then the synchro ring and the clutch gear keep relative static in the direction of rotation when their cones of friction contact with each other as the dynamic frictional torque on the cones equals zero, that is, $T_{s y n}=0$. As shown in Fig. $4 \mathrm{a}$, when the sleeve arrives at a tooth chamfer of the synchro ring, the $\Delta \theta_{s l v-s y n}=-\pi / 2 N$. Then, as shown in Fig. $4 \mathrm{~b}$, with the axial shifting force $F_{s}$, the sleeve pushes the synchro ring to rotate a half tooth thickness forward and passes through it, and then the sleeve begins to interact with the desired clutch gear.

(2) If the rotational speed of sleeve is greater than the desired clutch gear, that is, $\Delta \omega_{s l v-g r}>\varepsilon_{\omega}$, then as shown in Fig. 4a, a nonzero dynamic frictional torque $T_{s y n}$, opposite to the $\omega_{s l v}$, acts on the inner cone of synchro ring. The "lock-out" design of the synchro ring guarantees that the $T_{s y n}$ is greater than the tangential torque component yield from the shifting force $F_{s}$, and thus the lug boss of the synchro ring keeps contacting with the upper slot wall to lock out the sleeve, that is, $\Delta \theta_{s l v-s y n}=-\pi / 2 N$. Then, with the dynamic frictional torque $T_{s y n}$, the clutch gear is synchronized with the sleeve gradually. When they are synchronous, that is, $\Delta \omega_{s l v-g r}=0$, the $T_{s y n}$ disappears, and the "lock-out" to the sleeve is removed, and then as shown in Fig. 4b, the sleeve passes through the synchro ring after pushing it to rotate a half tooth thickness forward. 


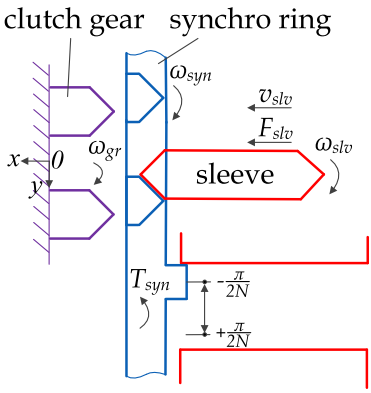

(a) Initial position

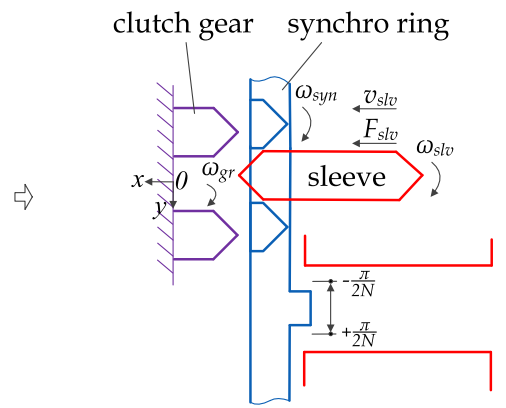

(b) Final position

Fig. 4 When $\left|\Delta \omega_{s l v-g r}\right| \leq \varepsilon_{\omega}$, there is no dynamic frictional torque on the inner cone of the synchro ring $\left(T_{s y n}=0\right)$, and the sleeve pushes the synchro ring to rotate a half tooth thickness forward and then passes through the synchro ring; when $\Delta \omega_{s l v-g r}>\varepsilon_{\omega}$, the synchro ring first locks out the sleeve, and the $\Delta \omega_{s l v-g r}$ approaches zero through a dynamic frictional torque $T_{s y n}$ on the cones of friction, and then the sleeve passes through the synchro ring

Fig. 5 When $\Delta \omega_{s l v-g r} \leq-\varepsilon_{\omega}$ and $\Delta \theta_{s l v-s y n} \in[-\pi / 2 N, 0]$, the synchro ring cannot lock out the sleeve when the sleeve teeth contact with its upper tooth chamfers, and then the sleeve passes through the synchro ring with a rotational speed difference

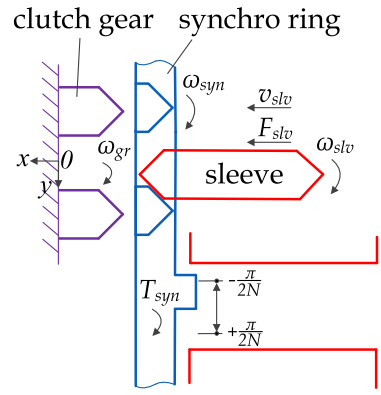

If the rotational speed of sleeve is smaller than the clutch gear, that is, $\Delta \omega_{s l v-g r}<-\varepsilon_{\omega}$, then as shown in Figs. 5, 6, 7, the $T_{s y n}$ and the $\omega_{s l v}$ have same direction of rotation, and the synchro ring rotates forward relative to the straight slot (sleeve). When the sleeve teeth contact with the tooth chamfers of the synchro ring, three cases are present according to their different relative tooth position $\Delta \theta_{s l v-s y n}$.

(3) If the $\Delta \theta_{s l v-s y n}$ is within $[-\pi / 2 N, 0]$, then as shown in Fig. 5, the synchro ring does not rotate a half tooth thickness forward and cannot lock out the sleeve. In this case, the sleeve goes through the synchro ring quickly after some impacts on its upper tooth chamfers and then collide with the clutch gear with a rotational speed difference, which results in tooth hitting seriously.

(4) If the synchro ring has rotated a half tooth thickness forward relative to the straight slot (sleeve) before the sleeve arrives at tooth chamfers of the synchro ring, that is, $\Delta \theta_{s l v-s y n} \in$ $(0, \pi / 2 N)$, then as shown in Fig. 6 , the synchro ring locks out the sleeve during the relative rotation. The sleeve stops its axial movement after some impacts on the lower tooth chamfers of the synchro ring, and then the $\Delta \omega_{s l v-g r}$ approaches zero with the dynamic frictional torque $T_{s y n}$ on the cones of friction. When the "lock-out" to the sleeve is removed, the sleeve passes through the synchro ring and then begins to interact with the clutch gear.

(5) If the synchro ring has rotated a tooth thickness forward relative to the straight slot (sleeve) and its lug boss arrives at the lower slot wall before the sleeve arrives at the synchro ring, that is, $\Delta \theta_{s l v-s y n}=\pi / 2 N$, then as shown in Fig. 7 , the synchro ring also locks 
Fig. 6 When $\Delta \omega_{s l v-g r} \leq-\varepsilon_{\omega}$ and $\Delta \theta_{s l v-s y n} \in(0, \pi / 2 N)$, the synchro ring locks out the sleeve during its rotation forward relative to the sleeve, and the sleeve cannot pass through the synchro ring until they are synchronous with clutch gear

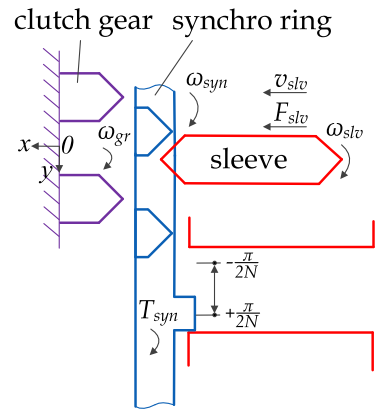

(a) Initial position

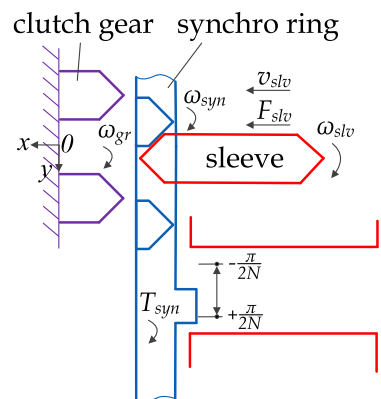

clutch gear synchro ring

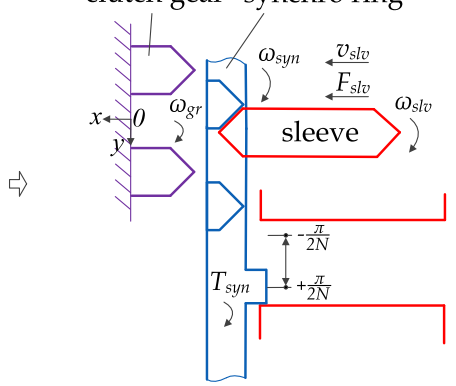

(b) Final position

Fig. 7 When $\Delta \omega_{s l v-g r} \leq-\varepsilon_{\omega}$ and $\Delta \theta_{s l v-s y n}=\pi / 2 N$, the synchro ring rotates a tooth thickness forward, and its lug boss arrives at the lower slot wall to lock out sleeve before the sleeve teeth contact with its lower tooth chamfers

out the sleeve. Since the rotational speeds of the sleeve and the synchro ring are the same $\left(\omega_{s l v}=\omega_{s y n}\right)$ when the sleeve teeth collide with the lower tooth chamfers of the synchro ring, the sleeve stops its axial movement after one impact, and then the $\Delta \omega_{s l v-g r}$ approaches zero with the dynamic frictional torque $T_{s y n}$.

\subsection{Interaction of sleeve and clutch gear}

The above interaction of the sleeve and synchro ring partially determines the interaction ways of the sleeve and the desired clutch gear through changing their relative rotational speed $\Delta \omega_{s l v-g r}$. In addition, as shown in Fig. 8, the relative tooth position of the sleeve and the clutch gear $\Delta \theta_{s l v-g r}$, where $\Delta \theta_{s l v-g r}=\theta_{s l v}-\theta_{g r}$, also determines their interaction way. As shown in Figs. 8a, 8b, 8d, 8e, the sleeve engages with the clutch gear after some impacts on obverse or reverse tooth chamfers. Of course, as shown in Fig. 8c, the splines of the sleeve may be aligned with the tooth space of the clutch gear exactly when the sleeve arrives at the clutch gear, and then the engagement proceeds smoothly without any impact at tooth chamfers.

\section{Modeling engaging process}

In this section, we develop a hybrid automaton (HA) model for the multimode engaging process. 


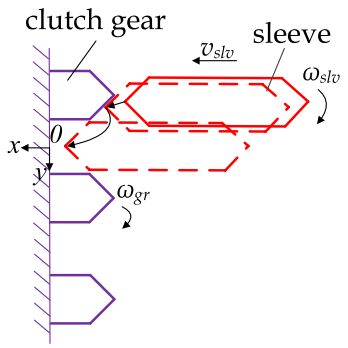

(a) Obverse contacting

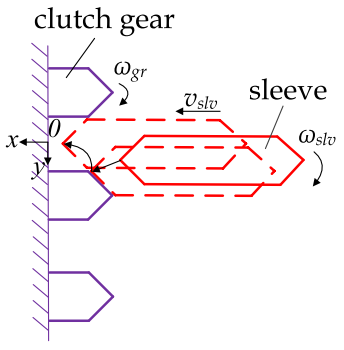

(d) Reverse contacting

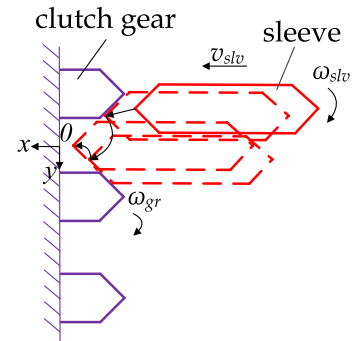

(b) Obverse contacting + Forwards + Reverse contacting

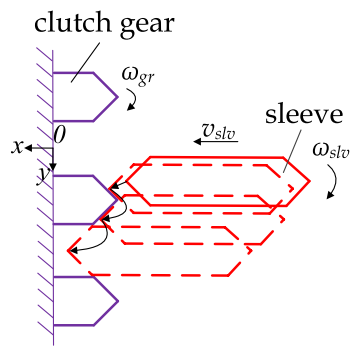

(e) Reverse contacting + Backwards + Obverse contacting

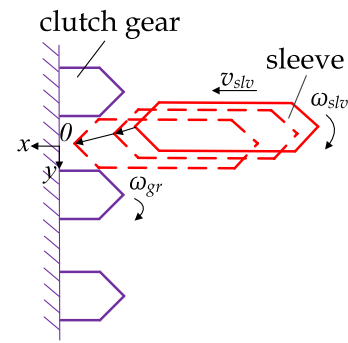

(c) Exact engaging

Fig. 8 Taking $\omega_{s l v}>\omega_{g r}$ as an example, five interaction ways of sleeve and clutch gear are present

Fig. 9 Six degrees of freedom are present in a synchronizer

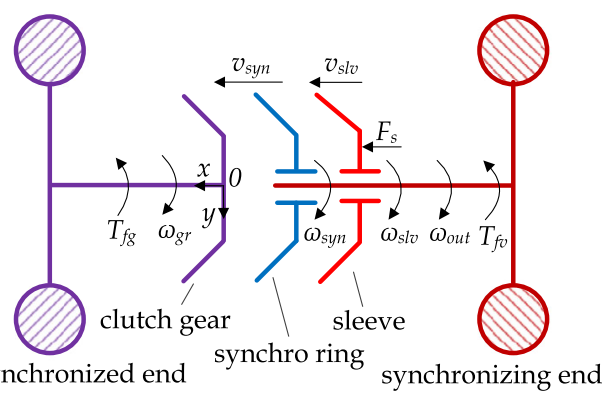

\subsection{Degrees of freedom analysis}

As shown in Fig. 9, we abstract the clutch gear, the synchro ring, the sleeve, and the transmission output shaft into four rigid bodies and build a two-dimensional plane coordinate on the clutch gear such that the four rigid bodies rotate around the $x$ axis.

First, at the synchronizing end, the transmission output shaft is connected with drive shafts, differential unit, and wheels mechanically, and they have a rotational degree of free$\operatorname{dom} \theta_{\text {out }}$. In addition, the sleeve also has a rotational degree of freedom $\theta_{s l v}$. Since the sleeve 
is connected with the output shaft through splines at a splined hub, they have the constraint $\theta_{\text {out }}=\theta_{s l v}$. The equivalent moment of inertia of these transmission parts is

$$
J_{\text {out }}=J_{s l v}+J_{h u b}+J_{o s h}+J_{v}
$$

where the $J_{s l v}$ is the moment of inertia of the sleeve, the $J_{h u b}$ is the moment of inertia of the splined hub, the $J_{\text {osh }}$ is the moment of inertia of the output shaft, and the $J_{v}$ is the equivalent moment of inertia of the drive shafts, the differential unit, the wheels, and the vehicle mass at the transmission output shaft. An equivalent drag torque $T_{f v}$ of vehicle resistances acts on the sleeve through these transmission parts.

Second, at the axial direction, with a shifting force $F_{s}$, the sleeve can move forward axially, and it has a translational degree of freedom $\left(x_{s l v}\right)$.

Third, the synchro ring has two degrees of freedom. First, since the synchro ring can rotate between the two sides of straight slot of splined hub, it has a rotational degree of freedom $\left(\theta_{s y n}\right)$. Second, with an axial pushing force from a sliding block, the synchro ring can move forward axially, and it has a translational degree of freedom $\left(x_{\text {syn }}\right)$.

Fourth, at the synchronized end, the rotational inertias include clutch plate $\left(J_{c l u}\right)$, transmission input shaft $\left(J_{i s h}\right)$, counter shaft $\left(J_{c s h}\right)$, and gearwheel and clutch gear $\left(J_{g r}\right)$. They are coupled together mechanically to rotate with an equivalent drag torque $T_{f g}$ at the clutch gear (including churning loss, wind drag, etc.), and their equivalent moment of inertia is

$$
J_{i n}=\left(J_{c l u}+J_{i s h}\right) \cdot i_{g 1}^{2} \cdot i_{g 2}^{2}+J_{c s h} \cdot i_{g 2}^{2}+J_{g r},
$$

where the $i_{g 1}$ represents the gear ratio between the transmission input shaft and the counter shaft, and the $i_{g 2}$ represents the gear ratio between the counter shaft and the gearwheel. We choose the rotation of the clutch gear $\left(\theta_{g r}\right)$ to represent this rotational degree of freedom.

Based on the above analysis, six degrees of freedom are present in the synchronizer, and they are: the axial movement $\left(x_{s l v}\right)$ and the rotation $\left(\theta_{s l v}\right)$ of sleeve, the axial movement $\left(x_{s y n}\right)$ and the rotation $\left(\theta_{\text {syn }}\right)$ of synchro ring, the rotation $\left(\theta_{\text {out }}\right)$ of output shaft, and the rotation $\left(\theta_{g r}\right)$ of clutch gear. A constraint is present as $\theta_{\text {out }}=\theta_{s l v}$.

\subsection{Hybrid automaton model}

Figure 10 shows an HA model that captures the trajectories of the sleeve, the synchro ring, and the clutch gear during the sleeve engaging with the clutch gear. Specifically, in the HA model, a discrete variable (loc) denotes the fifteen mechanical coupling modes of the three parts, which are defined as $l o c \in \mathcal{L}$, where $\mathcal{L} \triangleq\left\{\ell_{i}\right\}, i=1,2, \ldots, 15$. For each loc $\in \mathcal{L}$, an invariant set $\mathcal{I}_{i}$ formulates the mechanical constraint, and ten continuous variables denote the trajectories of the three parts, which are defined as $\mathbf{x} \triangleq\left\{\mathbf{x}_{1}, \mathbf{x}_{2}\right\}$, where $\mathbf{x}_{1} \triangleq\left\{v_{s l v}, \omega_{s l v}, v_{s y n}, \omega_{s y n}, \omega_{g r}\right\}$ and $\mathbf{x}_{2} \triangleq\left\{x_{s l v}, \theta_{s l v}, x_{s y n}, \theta_{s y n}, \theta_{g r}\right\}$. When $l o c=\ell_{i}$, the variables in $\mathbf{x}$ evolve according to the differential equations

$$
\left\{\begin{array}{l}
\dot{\mathbf{x}}_{1}=B_{i} \cdot \mathbf{u}+D_{1 i} \cdot f_{1}+D_{2 i} \cdot f_{2}+D_{3 i} \cdot f_{3}, \\
\dot{\mathbf{x}}_{2}=\mathbf{x}_{1},
\end{array}\right.
$$




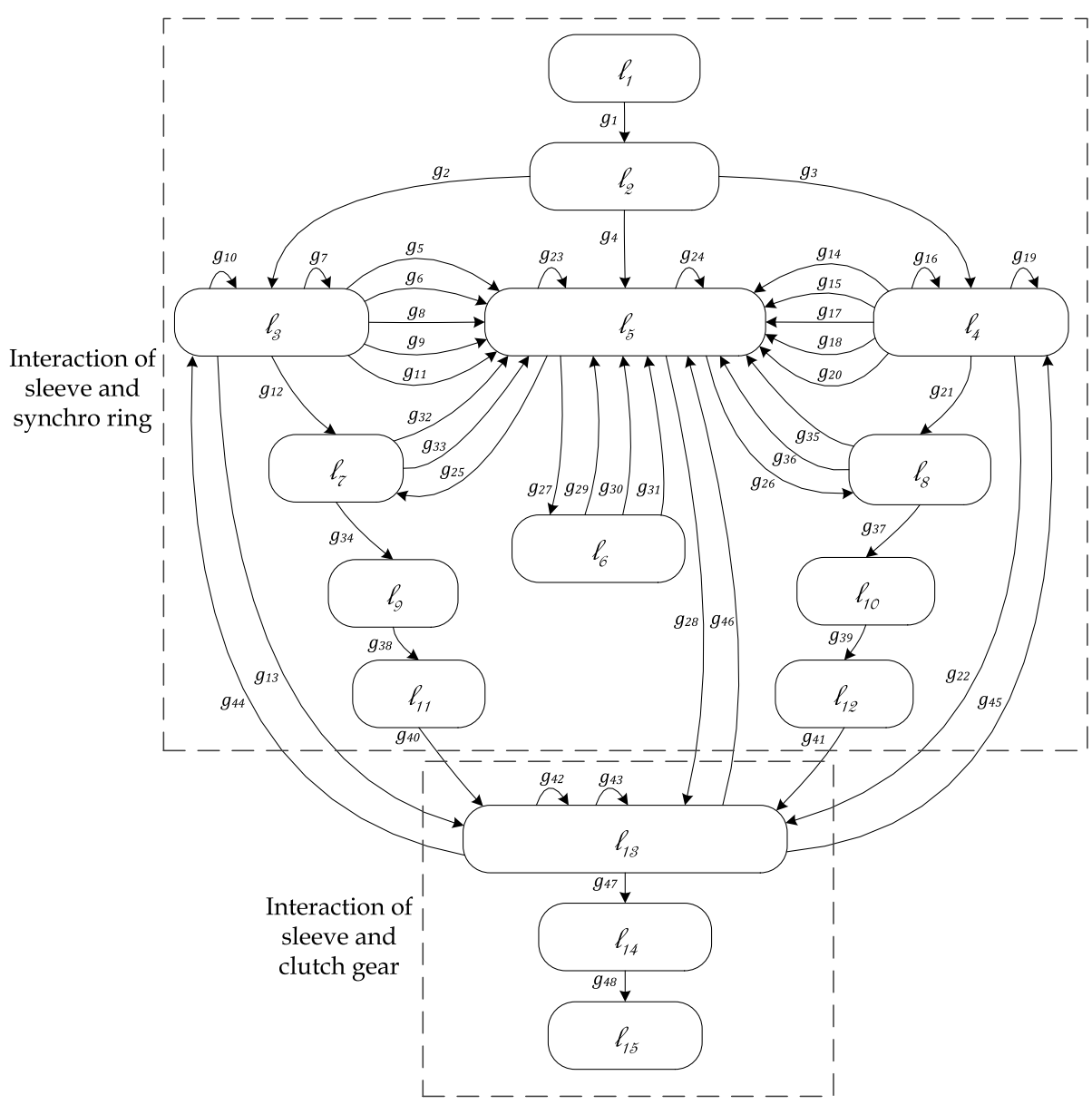

Fig. 10 The HA model of the engaging process contains two parts for the interactions of the sleeve, the synchro ring, and the clutch gear

where $B_{i}$ represents a $5 \times 1$ coefficient matrix, $D_{1 i}, D_{2 i}$, and $D_{3 i}$ represent three $5 \times 1$ coefficient matrices, respectively, $\mathbf{u}$ represents the model inputs and is defined as $\mathbf{u} \triangleq F_{s}$, and $f_{1}, f_{2}$, and $f_{3}$ represent three model disturbances defined as $f_{1} \triangleq T_{f g}, f_{2} \triangleq T_{f v}$, and $f_{3} \triangleq F_{f s}\left(F_{f s}\right.$ is a spring force; see Fig. $\left.3 \mathrm{~b}\right)$.

The HA model contains forty-eight guards to formulate the events that trigger the state discrete transitions of the variables, and they are defined as $G \triangleq\left\{g_{k}\right\}, k=1,2, \ldots, 48$. As shown in Fig. 10, when a $g_{k} \in G$ is triggered, at least one variable has a discrete state transition. According to a predefined reset map, the discrete variable loc may switch from $\ell_{i}$ to $\ell_{j}(i \neq j)$ for a switch of mechanical coupling mode, and the continuous variables in $\mathbf{x}$ may have jumps for an impact.

As a whole, the HA model of the engaging process contains two parts: loc from $\ell_{1}$ to $\ell_{12}$ models the interaction of the sleeve and the synchro ring, which covers their interaction ways in Sect. 2.1, and loc from $\ell_{13}$ to $\ell_{15}$ models the interaction of the sleeve and the clutch gear, which covers their interaction ways in Sect. 2.2. 


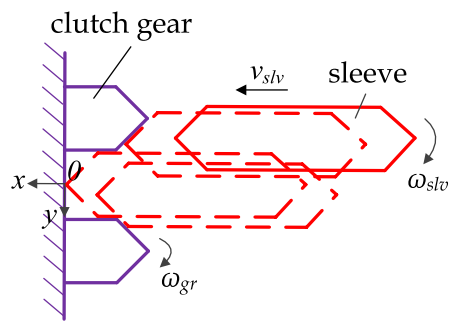

(a) Origin

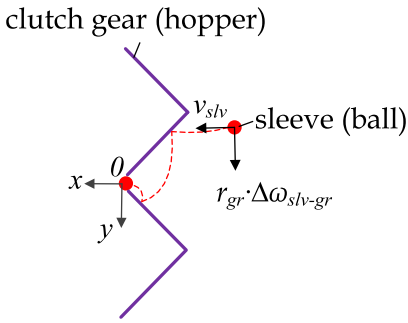

(b) Abstraction

Fig. 11 Clutch gear is captured by a "hopper" in 2D planes, and sleeve is modeled as a "ball"

\subsection{Impact model}

In this subsection, we develop an impact model to capture the discrete transitions of the axial and rotational speeds of the sleeve, the synchro ring, and the clutch gear when an impact happens on their tooth chamfers. A Poisson coefficient of restitution is used to quantify the energy ratio before and after impact. We take the impact on the tooth chamfers of the sleeve and the clutch gear as an example (Fig. 8) and then model the impact process with a Poisson coefficient of restitution. For the impact on the tooth chamfers of the sleeve and the synchro ring (Figs. 4 to 7), we could use the same method to model it.

As shown in Fig. 11, the clutch gear is captured by a "hopper" on the two-dimensional plane, and the sleeve is modeled as a "ball." We abstract the interaction process of sleeve and clutch gear into that the ball goes though the hopper after some impacts on the hopper walls. When the ball does not reach the hopper, the system dynamics are present in Appendix B.13. Once the ball arrives at the lower or upper wall of the hopper (that is, the sleeve contacts with the lower or upper tooth chamfers of the clutch gear), an impact happens such that the $v_{s l v}, \omega_{s l v}$, and $\omega_{g r}$ have state discrete transitions. In this example, the ball first arrives at the lower wall of the hopper, then arrives at the upper wall, and finally goes through the hopper opening after the two impacts.

(1) Impact on lower tooth chamfers. When the sleeve teeth collide with the lower tooth chamfers of the clutch gear, an impact happens, that is, the guard $g_{42}$ is triggered (Fig. 10). We divide the impact process into two phases-compression and recovery and ignore the effects of external forces and torques since the impact duration is too short. For the compression phase, an impact impulse $\Delta I_{1}$ makes the relative normal speed of sleeve and clutch gear be zero; for the recovery phase, another impact impulse $\Delta I_{2}\left(\Delta I_{2}=\zeta \cdot \Delta I_{1}\right)$ reverses their relative normal speed, where the $\zeta$ represents the Poisson coefficient of restitution between the tooth chamfers of sleeve and clutch gear. In this paper, the $\zeta$ equals 0.33 based on the materials of the sleeve and the clutch gear [12]. The compression equations are:

$$
\left\{\begin{array}{l}
v_{s l v}^{c}:=v_{s l v}-\frac{\sin \left(\alpha_{g r}\right) \cdot \Delta I_{1}}{m_{s l v}}, \\
\omega_{s l v}^{c}:=\omega_{s l v}+\frac{\cos \left(\alpha_{g r}\right) \cdot r_{g r} \cdot \Delta I_{1}}{J_{s y n}+J_{o u t}}, \\
\omega_{g r}^{c}:=\omega_{g r}-\frac{\cos \left(\alpha_{g r}\right) \cdot r_{g r} \cdot \Delta I_{1}}{J_{i n}}, \\
\cos \left(\alpha_{g r}\right) \cdot r_{g r} \cdot \omega_{g r}^{c}=\cos \left(\alpha_{g r}\right) \cdot r_{g r} \cdot \omega_{s l v}^{c}-\sin \left(\alpha_{g r}\right) \cdot v_{s l v}^{c},
\end{array}\right.
$$


where $v_{s l v}^{c}, \omega_{s l v}^{c}$, and $\omega_{g r}^{c}$ represent the reset values of $v_{s l v}, \omega_{s l v}$, and $\omega_{g r}$ after the compression phase. The recovery equations are:

$$
\left\{\begin{array}{l}
v_{s l v}^{r}:=v_{s l v}^{c}-\frac{\sin \left(\alpha_{g r}\right) \cdot \Delta I_{2}}{m_{s l v}}, \\
\omega_{s l v}^{r}:=\omega_{s l v}^{c}+\frac{\cos \left(\alpha_{g r}\right) \cdot r_{g r} \cdot \Delta I_{2}}{J_{s y n}+J_{o u t}}, \\
\omega_{g r}^{r}:=\omega_{g r}^{c}-\frac{\cos \left(\alpha_{g r}\right) \cdot r_{g r} \cdot \Delta I_{2}}{J_{i n}},
\end{array}\right.
$$

where $v_{s l v}^{r}, \omega_{s l v}^{r}$, and $\omega_{g r}^{r}$ represent the reset values of $v_{s l v}^{c}, \omega_{s l v}^{c}$, and $\omega_{g r}^{c}$ after the recovery phase. Solving the difference equations in (4) and (5), we can obtain the reset values of $v_{s l v}$, $\omega_{s l v}$, and $\omega_{g r}$ after an impact on the lower tooth chamfers of the clutch gear.

(2) Impact on upper tooth chamfers. When the sleeve teeth collide with the upper tooth chamfers of the clutch gear, the guard $g_{43}$ is triggered (Fig. 10). Similarly with the impact on lower tooth chamfers, compression and recovery phases are also present, and we could readily obtain the reset values of $v_{s l v}, \omega_{s l v}$, and $\omega_{g r}$ through solving the compression and the recovery equations of the impact.

\subsection{Model integration}

In this subsection, we integrate the HA model in Fig. 10 through specifying the mode and state discrete transitions for the two interaction phases of the engaging process. The invariant sets and the guards of the two phases are listed in Tables 1 and 2, respectively. The equations describing the multibody dynamics of each mode are displayed in Appendix B.

\subsubsection{Specification of the interaction of sleeve and synchro ring}

In $\ell_{1}$, the sleeve is at neutral gear and moves axially with a shifting force $F_{s}$ on it. When sliding block contacts with the left end face of synchro ring (Fig. 3), an impact happens to make them move forward axially together. That is, when the $g_{1}$ is triggered, the loc switches from $\ell_{1}$ to $\ell_{2}$, and both $v_{s l v}$ and $v_{s y n}$ have discrete transitions as

$$
v_{s l v}^{\prime}=v_{s y n}^{\prime}:=\frac{\left(m_{s l v}+m_{s l i d}\right) \cdot v_{s l v}}{m_{s l v}+m_{s l i d}+m_{s y n}} .
$$

In $\ell_{2}$, the sleeve, the sliding block, and the synchro ring move forward axially. When the inner cone of synchro ring contacts with the outer cone of clutch gear (Fig. 3), an impact happens to stop the axial movement of synchro ring, and then $v_{s y n}^{\prime}:=0$. As discussed in Sect. 2.1, the $l o c$ switches in accordance with the $\Delta \omega_{s l v-g r}$. (a) When the $g_{2}$ is triggered, the loc switches from $\ell_{2}$ to $\ell_{3}$. (b) When the $g_{3}$ is triggered, the loc switches from $\ell_{2}$ to $\ell_{4}$. (c) When the $g_{4}$ is triggered, the loc switches from $\ell_{2}$ to $\ell_{5}$.

In $\ell_{3}$, at the rotational direction, the synchro ring rotates forward relative to the straight slot (sleeve) as in cases 3-5 in Sect. 2.1, and at the axial direction, the sleeve moves forward axially. State discrete transition happens with five cases. (a) When the sleeve teeth contact with the upper tooth chamfers of synchro ring, an impact happens as in case 3 in Sect. 2.1. The minimum impact impulse between the spline chamfers of the sleeve and the synchro ring to synchronize the synchro ring and clutch gear is $\Delta I_{3}$. If the impact impulse on the splined chamfers is greater than the $\Delta I_{3}$, then $g_{5}$ or $g_{6}$ (depending on after compression 
Table 1 The invariant sets and the guards of the interaction of sleeve and synchro ring*

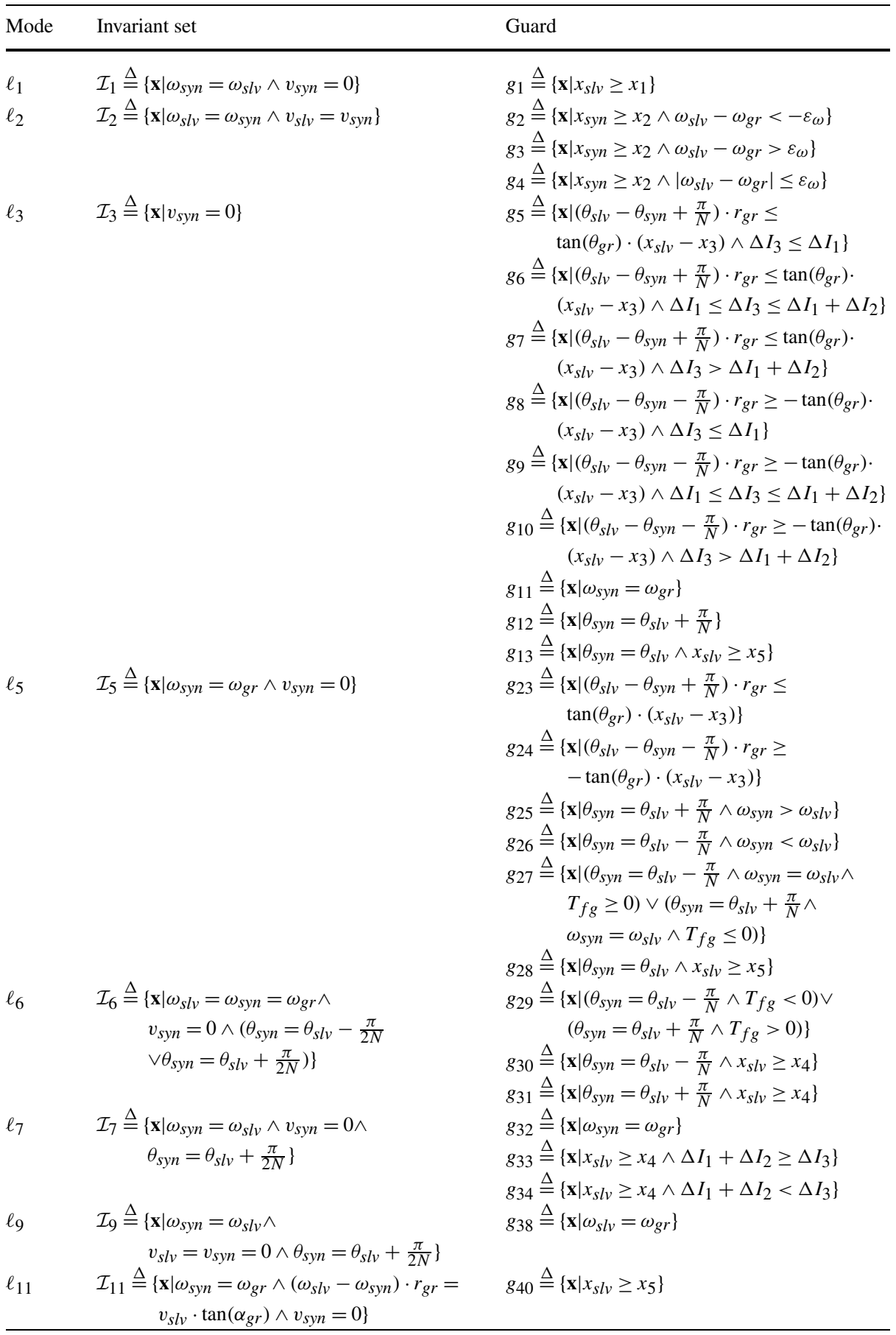

*With reference to the $\ell_{3}, \ell_{7}, \ell_{9}$, and $\ell_{11}$, we could readily obtain the invariant sets and the guards of $\ell_{4}, \ell_{8}$, $\ell_{10}$, and $\ell_{12}$ 
Table 2 The invariant sets and the guards of the interaction of sleeve and clutch gear

\begin{tabular}{|c|c|c|}
\hline Mode & Invariant set & Guard \\
\hline$\ell_{13}$ & $\begin{array}{c}\mathcal{I}_{13} \triangleq\left\{\mathbf{x} \mid \omega_{s l v}=\omega_{s y n} \wedge v_{s y n}=0 \wedge\right. \\
\left.\theta_{s l v}=\theta_{s y n}\right\}\end{array}$ & $\begin{array}{l}g_{42} \triangleq\left\{\mathbf{x} \mid\left(\theta_{s l v}-\theta_{g r}+\frac{\pi}{N}\right) \cdot r_{g r} \leq \tan \left(\theta_{g r}\right) \cdot\left(x_{s l v}-x_{6}\right)\right\} \\
g_{43} \triangleq\left\{\mathbf{x} \mid\left(\theta_{s l v}-\theta_{g r}-\frac{\pi}{N}\right) \cdot r_{g r} \geq-\tan \left(\theta_{g r}\right) \cdot\left(x_{s l v}-x_{6}\right)\right\} \\
g_{44} \triangleq\left\{\mathbf{x} \mid x_{s l v} \leq x_{5} \wedge \omega_{s l v}-\omega_{g r}<-\varepsilon_{\omega}\right\} \\
g_{45} \triangleq\left\{\mathbf{x} \mid x_{s l v} \leq x_{5} \wedge \omega_{s l v}-\omega_{g r}>\varepsilon_{\omega}\right\} \\
g_{46} \triangleq\left\{\mathbf{x}\left|x_{s l v} \leq x_{5} \wedge\right| \omega_{s l v}-\omega_{g r} \mid \leq \varepsilon_{\omega}\right\} \\
g_{47} \triangleq\left\{\mathbf{x} \mid x_{s l v} \geq x_{7}\right\}\end{array}$ \\
\hline$\ell_{14}$ & $\begin{array}{r}\mathcal{I}_{14} \triangleq\left\{\mathbf{x} \mid \omega_{s l v}=\omega_{s y n}=\omega_{g r} \wedge\right. \\
\left.v_{s y n}=0 \wedge \theta_{s l v}=\theta_{s y n}\right\}\end{array}$ & $g_{48} \triangleq\left\{\mathbf{x} \mid x_{s l v} \geq x_{8}\right\}$ \\
\hline$\ell_{15}$ & $\begin{array}{c}\mathcal{I}_{15} \triangleq\left\{\mathbf{x} \mid \omega_{s l v}=\omega_{s y n}=\omega_{g r} \wedge\right. \\
v_{s l v}=v_{s y n}=0 \wedge \\
\left.\theta_{s l v}=\theta_{s y n}\right\}\end{array}$ & \\
\hline
\end{tabular}

phase or after recovery phase) is triggered, and the loc switches to $\ell_{5}$; if not, $g_{7}$ is triggered, and the loc remains unchanged. (b) When the sleeve teeth contact with the lower tooth chamfers of synchro ring, an impact happens as in case 4 in Sect. 2.1, and $g_{8}, g_{9}$, or $g_{10}$ is triggered also depending on the impact impulse. (c) When the synchro ring and clutch gear are synchronous before the sleeve teeth arrive at a splined chamfer of the synchro ring, the dynamic frictional torque $T_{s y n}$ disappears, but a pushing force $F_{s y n}$ from sliding block still acts on the synchro ring, and then the synchro ring and the clutch gear are coupled together to rotate with a static frictional torque $T_{s y n}^{*}$ instead. That is, when $g_{11}$ is triggered, the $l o c$ switches from $\ell_{3}$ to $\ell_{5}$, but the variables in $\mathbf{x}$ keep unchanged. (d) When the lug boss of the synchro ring arrives at the lower wall of straight slot, an impact happens to stop the rotation of the synchro ring relative to the straight slot (sleeve) as in case 5 in Sect. 2.1. That is, when $g_{12}$ is triggered, the loc switches from $\ell_{3}$ to $\ell_{7}$, and $\omega_{s y n}^{\prime}:=\omega_{s l v}$. (e) As a special case of case 3 in Sect. 2.1, the sleeve engages with the synchro ring exactly if $\Delta \theta_{s l v-s y n}=0$ when the sleeve arrives at the synchro ring (Fig. 4b), no impact happens to block the axial movement of the sleeve but an impact happens at the rotational direction to synchronize the synchro ring with the sleeve, and then they are coupled together to rotate. That is, when $g_{13}$ is triggered, the loc switches from $\ell_{3}$ to $\ell_{13}$, and $\omega_{s y n}^{\prime}:=\omega_{s l v}$.

In $\ell_{4}$, at the rotational direction, the synchro ring rotates backward relative to the straight slot (sleeve) as in case 2 in Sect. 2.1, and at the axial direction, the sleeve moves forward axially. With reference to the $\ell_{3}$, we could readily obtain the invariant set $\mathcal{I}_{4}$ and state discrete transitions from $g_{14}$ to $g_{22}$.

In $\ell_{5}$, as in case 1 in Sect. 2.1, the synchro ring and clutch gear are synchronous and coupled together to rotate with a static frictional torque $T_{s y n}^{*}$ on their cones. State discrete transition happens with six cases. (a) When the sleeve teeth contact with the upper tooth chamfers of the synchro ring (Fig. 4a), an impact happens, and the synchro ring and the clutch gear keep synchronous after the impact due to the "lock-out" design of synchro ring. That is, when $g_{23}$ is triggered, the loc keeps in $\ell_{5}$. (b) Also, when the sleeve teeth contact with the lower tooth chamfers of the synchro ring, $g_{24}$ is triggered, and also the loc keeps in $\ell_{5}$. (c) When the lug boss arrives at lower slot wall after rotating a tooth thickness forward relative to the sleeve (Fig. 7a), an impact happens to stop the rotation of the synchro ring relative to the sleeve. That is, when $g_{25}$ is triggered, the loc switches from $\ell_{5}$ to $\ell_{7}$, and 
$\omega_{s y n}^{\prime}:=\omega_{s l v}$. (d) Also, when the lug boss arrives at upper slot wall after rotating a tooth thickness backward relative to the sleeve, $g_{26}$ is triggered, the loc switches from $\ell_{5}$ to $\ell_{8}$, and $\omega_{s y n}^{\prime}:=\omega_{s l v}$. (e) If the synchro ring and the sleeve are synchronous when the lug boss is at upper or lower slot wall exactly, then the sleeve, the synchro ring, and the clutch gear keep relatively static at the rotational direction. That is, when the $g_{27}$ is triggered, the $l o c$ switches from $\ell_{5}$ to $\ell_{6}$, but the variables in $\mathbf{x}$ keep unchanged. (f) Also, if $\Delta \theta_{s l v-s y n}=0$ when the sleeve arrives at the synchro ring, $g_{28}$ is triggered, the $l o c$ switches from $\ell_{5}$ to $\ell_{13}$, and $\omega_{\text {syn }}^{\prime}:=\omega_{s l v}$.

In $\ell_{6}$, as a special case of case 1 in Sect. 2.1, the lug boss stops at a slot wall, and the sleeve, the synchro ring, and the clutch gear are coupled together to rotate. State discrete transition happens with three cases. (a) When the pressure between the lug boss and a slot wall disappears, the lug boss separates from the slot wall. That is, when $g_{29}$ is triggered, the loc switches from $\ell_{6}$ to $\ell_{5}$, but the variables in $\mathbf{x}$ keep unchanged. (b) As shown in Fig. $4 \mathrm{a}$, an impact happens to accelerate the rotation of the synchro ring and the clutch gear when the sleeve teeth contact with the upper tooth chamfers of the synchro ring, and then the lug boss separates from the upper slot wall. That is, when $g_{30}$ is triggered, the loc switches from $\ell_{6}$ to $\ell_{5}$. (c) As shown in Fig. 7b, an impact happens to slow down the rotation of the synchro ring and the clutch gear when the sleeve teeth contact with the lower tooth chamfers of the synchro ring; also, the lug boss separates from the lower slot wall. That is, when $g_{31}$ is triggered, the $l o c$ switches from $\ell_{6}$ to $\ell_{5}$.

In $\ell_{7}$, as in case 5 in Sect. 2.1, at the rotational direction, the lug boss of the synchro ring keeps contacting with the lower slot wall, and the rotational speed of the clutch gear is greater than the synchro ring; at the axial direction, the sleeve moves forward axially. State discrete transition happens with two cases. (a) When the synchro ring and the clutch gear are synchronous, the dynamic frictional torque $T_{s y n}$ disappears, and then they are coupled together to rotate with a static frictional torque $T_{s y n}^{*}$ instead. That is, when $g_{32}$ is triggered, the loc switches from $\ell_{7}$ to $\ell_{5}$, but the variables in $\mathbf{x}$ keep unchanged. (b) As shown in Fig. $7 b$, when the sleeve teeth contact with the lower tooth chamfers of the synchro ring, an impact happens to slow down the rotation of the clutch gear. If the impact impulse on the splined chamfers is big enough to synchronize the synchro ring and the clutch gear after the impact, then $g_{33}$ is triggered, and the $l o c$ switches from $\ell_{7}$ to $\ell_{5}$; if not, $g_{34}$ is triggered, the loc switches from $\ell_{7}$ to $\ell_{9}$, and $v_{s l v}^{\prime}:=0$.

In $\ell_{8}$, as in case 2 in Sect. 2.1, the lug boss of the synchro ring keeps contacting with the upper slot wall, and the rotational speed of the clutch gear is smaller than the synchro ring. With reference to the $\ell_{7}$, we can readily obtain the state discrete transitions from $g_{35}$ to $g_{37}$.

In $\ell_{9}$, at the rotational direction, the lug boss of the synchro ring keeps contacting with the lower slot wall; at the axial direction, sleeve teeth keep contacting with the lower tooth chamfers of the synchro ring to synchronize the clutch gear with the sleeve (Fig. 7b). When the sleeve, the synchro ring, and the clutch gear are synchronous, the synchro ring and clutch gear keep relatively static, and the sleeve moves forward axially. That is, when $g_{38}$ is triggered, the $l o c$ switches from $\ell_{9}$ to $\ell_{11}$, but the variables in $\mathbf{x}$ keep unchanged.

In $\ell_{10}$, the lug boss keeps contacting with the upper slot wall, and the sleeve teeth keep contacting with the upper tooth chamfers of the synchro ring to synchronize the clutch gear with the sleeve (Fig. 4a). With reference to the $\ell_{9}$, we can readily obtain the state discrete transition of $g_{39}$.

In $\ell_{11}$, the sleeve moves forward axially to push the synchro ring and the clutch gear to rotate a half tooth thickness backward relative to the sleeve. When the sleeve engages with the synchro ring (Fig. 4b), an impact happens at the rotational direction to synchronize the synchro ring with the sleeve, and then they are coupled together to rotate. That is, when $g_{40}$ is triggered, the loc switches from $\ell_{11}$ to $\ell_{13}$, and $\omega_{s y n}^{\prime}:=\omega_{s l v}$. 
In $\ell_{12}$, the sleeve moves forward axially to push the synchro ring and the clutch gear to rotate a half tooth thickness forward relative to the sleeve (Fig. 4). With reference to the $\ell_{11}$, we can readily obtain the state discrete transition of $g_{41}$.

\subsubsection{Specification of the interaction of sleeve and clutch gear}

In $\ell_{13}$, at the rotational direction, the sleeve engages with the synchro ring, and at the axial direction, the sleeve moves forward axially. State discrete transition happens with six cases. (a) When the sleeve teeth contact with the upper tooth chamfers of the clutch gear, an impact happens as in Fig. $8 \mathrm{~d}$ or $8 \mathrm{e}$. That is, when $g_{42}$ is triggered, the loc does not switch, but $v_{s l v}, \omega_{s l v}$, and $\omega_{g r}$ have discrete state transitions according to the impact model in Sect. 3.3. (b) When the sleeve teeth contact with the lower tooth chamfers of the clutch gear (Figs. 8a and $8 \mathrm{~b}), g_{43}$ is triggered, the $l o c$ does not switch, but $v_{s l v}, \omega_{s l v}$, and $\omega_{g r}$ have discrete state transitions. If an impact on the tooth chamfers of the sleeve and the clutch gear is too high such that the sleeve disengages from the synchro ring after the impact, then the sleeve resumes an interaction with the synchro ring. Since the rotational speeds of the sleeve and the clutch gear are uncertain when the disengagement happens, the loc switches in accordance with the $\Delta \omega_{s l v-g r}$; (c) When $g_{44}$ is triggered, the loc switches from $\ell_{13}$ to $\ell_{3}$; (d) When $g_{45}$ is triggered, the loc switches from $\ell_{13}$ to $\ell_{4}$; (e) When $g_{46}$ is triggered, the loc switches from $\ell_{13}$ to $\ell_{5}$; (f) When the sleeve engages with the clutch gear after some impacts on the splined chamfers (Figs. 8a, 8b, 8d, 8e) or without any impact (Fig. 8c), an impact happens at the rotational direction to remove the rotational speed difference of the clutch gear and the sleeve. That is, when $g_{47}$ is triggered, the $l o c$ switches from $\ell_{13}$ to $\ell_{14}$, and $\omega_{g r}^{\prime}:=\omega_{s l v}$.

In $\ell_{14}$, at the rotational direction, the sleeve engages with clutch gear, and at the axial direction, the sleeve moves forward axially. When the sleeve arrives at the self-locking position of the desired clutch gear and engages with the clutch gear completely (Fig. 11a), an impact happens to stop its axial movement. That is, when $g_{48}$ is triggered, the loc switches from $\ell_{14}$ to $\ell_{15}$, and $v_{s l v}^{\prime}:=0$.

In $\ell_{15}$, at the rotational direction, the sleeve engages with the clutch gear, and at the axial direction, the sleeve keeps static.

\section{Simulation and characteristic analysis}

The HA model enables the characteristic analysis of the engaging process. In order to evaluate the performance of the engaging, we take engaging duration $t_{\text {sum }}$ and maximum impact impulse $I_{\max }$ as evaluation indexes [1,8]. We take the engaging process when $\omega_{s l v}=46.4(\mathrm{rad} / \mathrm{s})$ and $\omega_{g r}=81.6(\mathrm{rad} / \mathrm{s})$ as examples, and we solve the HA model in MATLAB. For each calculation, it takes about 130 seconds on a PC with an i5-3210 CPU and a 4 GB RAM. First, we illustrate the two interaction phases in Sect. 2 through showing an engaging process with a shifting force $F_{s}=100(\mathrm{~N})$. Next, we carry out more simulation experiments with different relative tooth positions of sleeve and clutch gear $\left(\Delta \theta_{s l v-g r}\right)$ and then analyze its effect on the $t_{\text {sum }}$ and $I_{\max }$. Finally, we increase the shifting force $F_{s}$ and analyze its effect on the $t_{\text {sum }}$ and $I_{\max }$.

\subsection{Discussion of the two interaction phases}

An engagement happens when the $\Delta \theta_{s l v-g r}=0.017$ (rad). The switching sequence of the location of the HA model is

$$
\ell_{1} \rightarrow \ell_{2} \rightarrow \ell_{3} \rightarrow \ell_{5} \rightarrow \ell_{7} \rightarrow \ell_{9} \rightarrow \ell_{11} \rightarrow \ell_{13} \rightarrow \ell_{14} \rightarrow \ell_{15},
$$




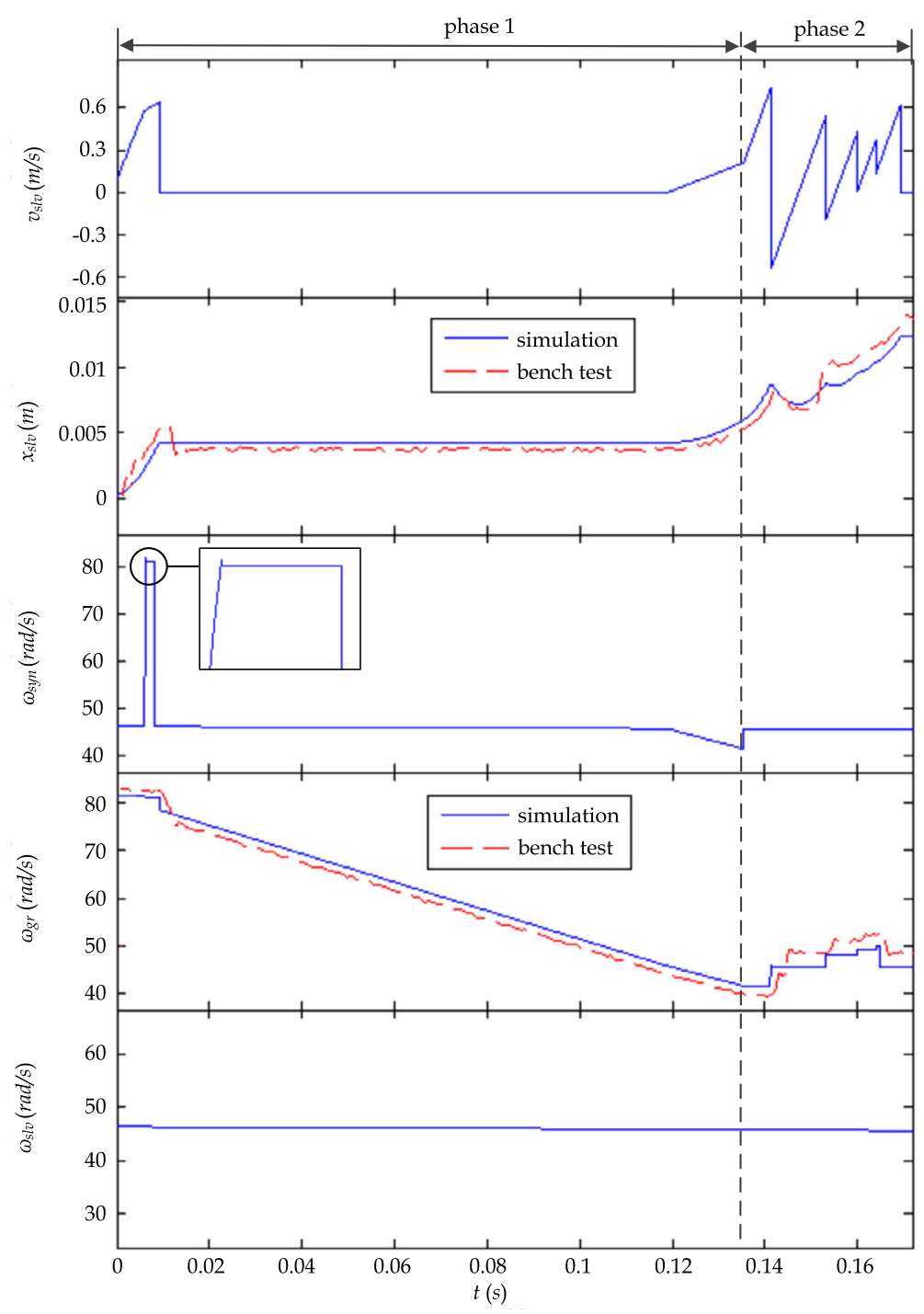

Fig. 12 The states of the HA model variables $\left(v_{s l v}, x_{s l v}, \omega_{s y n}, \omega_{g r}\right.$, and $\left.\omega_{s l v}\right)$ change as the engaging proceeds. The "phase 1" represents the interaction process of sleeve and synchro ring; the "phase 2" represents the interaction process of sleeve and clutch gear

and as shown in Fig. 12, the states of the speeds $\left(v_{s l v}, \omega_{s y n}, \omega_{g r}\right.$, and $\left.\omega_{s l v}\right)$ change both continuously and discretely, whereas the axial position of sleeve $\left(x_{s l v}\right)$ evolves only continuously.

The interaction process of sleeve and synchro ring belongs to the fifth case in Sect. 2.1 and lasts for $0.1354 \mathrm{~s}$. The synchro ring starts to move forward axially together with the sleeve when the left end face of the sliding block contacts with the left end face of the synchro ring at $0.0059 \mathrm{~s}$. The inner cone of the synchro ring arrives at the outer cone of 
Fig. 13 A test bench for the engaging process of mechanical transmission

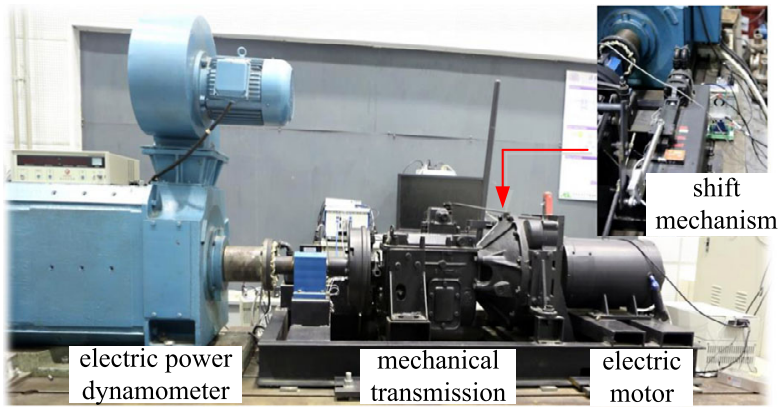

the clutch gear at $0.0061 \mathrm{~s}$, and the synchro ring rotates forward relative to the sleeve for $\omega_{s l v}<\omega_{g r}$ (see the $\omega_{s y n}$ in Fig. 12). Note that we zoom in the vertices of the curve for illustrating the gradual rise and the sudden drop of the $\omega_{s y n}$. The lug boss of the synchro ring arrives at the lower slot wall at $0.0082 \mathrm{~s}$, and then the $\omega_{s y n}$ recovers to be consistent with the sleeve through an impact with the lower slot wall. The sleeve arrives at the lower tooth chamfer of the synchro ring at $0.0093 \mathrm{~s}$, and an impact happens to drop the $v_{s l v}$ from 0.64 to $0 \mathrm{~m} / \mathrm{s}$, and then the sleeve stops its axial movement instantaneously. With the shifting force $F_{s}$ on the sleeve, the rotational speed of the clutch gear $\omega_{g r}$ decreases gradually while the rotational speed of the sleeve $\omega_{s l v}$ only has a minor decrease for the larger moment of inertia $J_{\text {out }}$ at the transmission output shaft. The $\omega_{g r}$ is synchronized with the $\omega_{s l v}$ at $0.1192 \mathrm{~s}$, and then the sleeve pushes the synchro ring and the clutch gear to rotate a half tooth thickness backward, which results in small decreases of the $\omega_{g r}$ and the $\omega_{s l v}$. Finally, the sleeve engages with the synchro ring at $0.1354 \mathrm{~s}$.

The interaction process of sleeve and clutch gear belongs to the fourth case in Sect. 2.2 and lasts for $0.0339 \mathrm{~s}$. At $0.1413 \mathrm{~s}$, the sleeve teeth contact with the upper tooth chamfers of the clutch gear. As shown in Fig. 12, the $\omega_{s l v}$ is greater than the $\omega_{g r}$ at this moment. As a result, the upper tooth chamfer is at the direction of reverse contact, and the impact makes the $v_{s l v}$ drop from 0.75 to $-0.54 \mathrm{~m} / \mathrm{s}$, and the $\omega_{g r}$ rise from 41.57 to $45.75 \mathrm{rad} / \mathrm{s}$. Note that the $\omega_{g r}$ is (almost) consistent with the $\omega_{s l v}$ after the impact, but as their splined tooth position is misaligned ( $\Delta \theta_{s l v-g r} \neq 0$ ), another three impacts happen to realign their splined tooth. Finally, the sleeve engages with the clutch gear at $0.1652 \mathrm{~s}$.

The engaging duration $t_{\text {sum }}$ of this example is $0.1693 \mathrm{~s}$, and the maximum impact impulse $I_{\max }$ is $1.41(\mathrm{~kg} \mathrm{~m} / \mathrm{s})$, which happens when the sleeve teeth contact with the upper tooth chamfers of the clutch gear for the first time.

A test bench for the engaging process of mechanical transmission is shown in Fig. 13. At the input end of the transmission, an electric motor is included to regulate the rotational speed of the clutch gear; at the output end of the transmission, an electric power dynamometer is included to regulate the rotational speed of the sleeve. In addition, a shift mechanism is included to output the shift force $F_{s}$ to push the sleeve forward. We carry out an engaging bench test with the same inputs and initial states as the simulation, and the $x_{s l v}$ and $\omega_{g r}$ are shown in Fig. 12. The change curves of the bench test are highly consistent with the simulation, and the result validates the high fidelity of the HA model.

\subsection{Discussion of the relative tooth position of sleeve and clutch gear}

As discussed in Sects. 2.2 and 4.1, the $\Delta \theta_{s l v-g r}$ has an effect on the interaction process of sleeve and clutch gear. Since the distribution of the $\Delta \theta_{s l v-g r}$ is approximately uniform, 
Fig. 14 The $t_{\text {sum }}$ and the $I_{\max }$ vary over the $\Delta \theta_{s l v-g r}$, and the interaction way of sleeve and clutch gear also switches between the five cases in Sect. 2.2

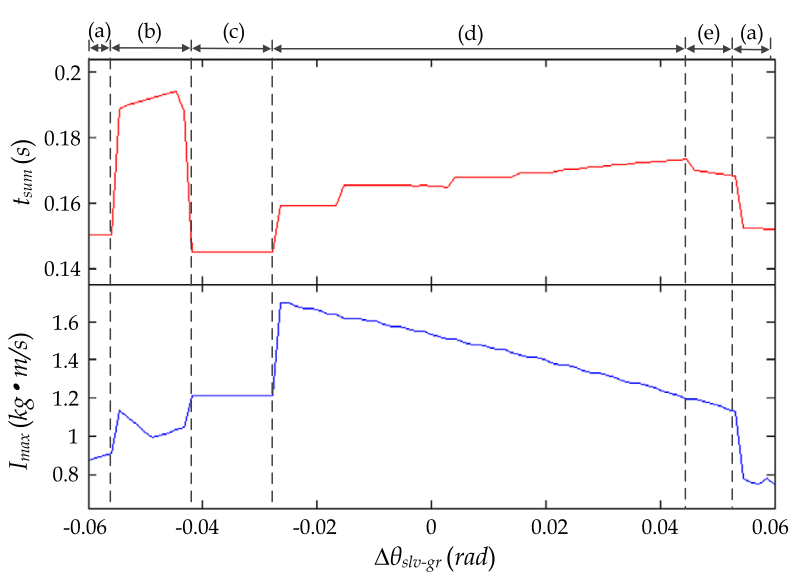

we discretize its feasible set $\left[-\frac{\pi}{N},+\frac{\pi}{N}\right]$ into 100 points uniformly, and then, we carry out simulations with each $\Delta \theta_{s l v-g r}$.

As shown in Fig. 14, when the $\Delta \theta_{s l v-g r}$ increases from -0.06 to $0.06 \mathrm{rad}$, the five interaction ways of sleeve and clutch gear discussed in Sect. 2.2 are present. When $\Delta \theta_{s l v-g r} \in$ $[-0.056,-0.043](\mathrm{rad})$, the interaction process belongs to the second case, and the sleeve teeth first contact with the lower tooth chamfers of the clutch gear. Note that as discussed in Sect. 4.1, the $\omega_{s l v}$ is greater than the $\omega_{g r}$ before the sleeve arrives at the clutch gear, then the impact happens at the obverse direction and decreases the $\omega_{g r}$ further, that is, the $\Delta \omega_{s l v-g r}$ increases. Next, when the sleeve arrives at the upper tooth chamfers, it has an impact at the reverse direction, and the enlarged $\Delta \omega_{s l v-g r}$ makes the sleeve move backwards after the impact and spend more time to reach the tooth spaces of the clutch gear, which results in a longer engaging duration $t_{s u m}$. For the third case, since the $\omega_{s l v}$ is greater than the $\omega_{g r}$, the splined teeth of the sleeve would align with the tooth spaces of the clutch gear exactly when the sleeve is at some backward tooth positions of the clutch gear initially, that is, $\Delta \theta_{s l v-g r} \in[-0.042,-0.028]$ (rad). The fourth interaction case occupies the largest proportion $(60 \%)$ of the $\Delta \theta_{s l v-g r}$, and as the impact always happens at the reverse direction, the $I_{\text {max }}$ is larger than in other cases.

\subsection{Discussion of the shifting force}

In this subsection, we increase the shifting force $F_{s}$ from 100 to $300 \mathrm{~N}$ and then observe the change rules of the $t_{s u m}$ and $I_{\max }$. As discussed in Sect. 4.2, the $\Delta \theta_{s l v-g r}$ has an effect on the $t_{\text {sum }}$ and $I_{\max }$. For the soundness of the analysis, we carry out simulations with different $\Delta \theta_{s l v-g r}$ under each $F_{s}$ and then choose the maximum, minimum, and mean values of the $t_{\text {sum }}$ and the $I_{\max }$ for each $F_{s}$.

When the $F_{s}$ increases, the sleeve moves forward axially with a higher acceleration, which makes the mean value of the $t_{\text {sum }}$ decrease and the $I_{\max }$ increase (Fig. 15). Note that the maximum and minimum values of the $t_{\text {sum }}$ and $I_{\max }$ have same trend with the mean value, although the $\theta_{s l v-g r}$ makes them different under a certain $F_{s}$.

The generating mechanism of the maximum impact switches when the $F_{s}$ increases. When $F_{s}<200 \mathrm{~N}$, the interaction of the sleeve and the synchro ring belongs to the fifth case in Sect. 2.1. As the rotational speed of the synchro ring is consistent with the sleeve $\left(\Delta \omega_{s l v-s y n}=0\right)$ in this case, the impact impulse between them is relatively low, and the maximum impact happens when the sleeve interacts with the clutch gear, which is affected 
Fig. 15 The $t_{\text {sum }}$ and $I_{\max }$ vary over the shifting force $F_{S}$

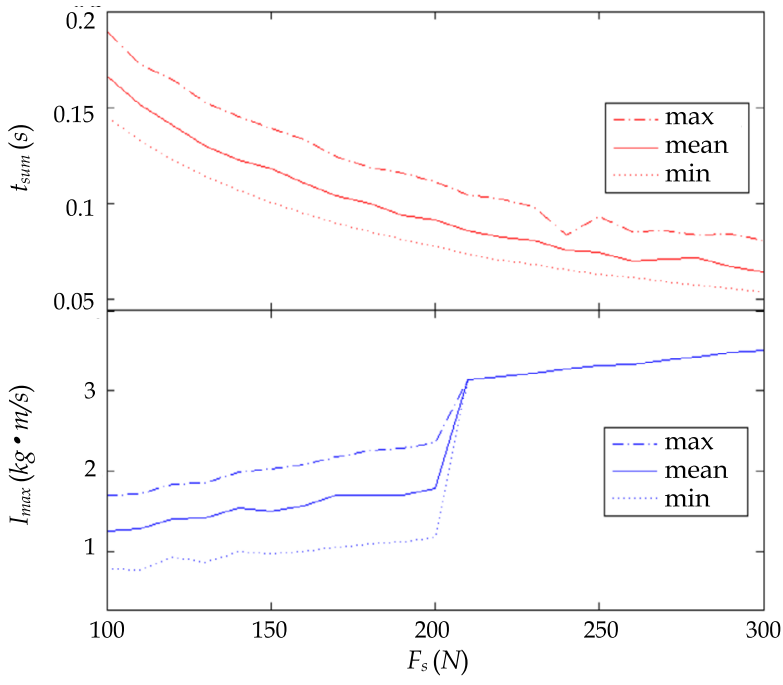

by the uncertain $\theta_{s l v-g r}$. As a result, the maximum, minimum, and mean values of the $I_{\max }$ are inconsistent when $F_{s}<200 \mathrm{~N}$. However, the three values of the $I_{\max }$ become consistent when $F_{s} \geq 200 \mathrm{~N}$. In this case, the interaction of the sleeve and the synchro ring belongs to the fourth case in Sect. 2.1. Since the rotational speed of the synchro ring is greater than the sleeve when the sleeve teeth arrive at the lower tooth chamfers of the synchro ring, an impact happens at the reverse direction, and the impact impulse is higher than the interaction phase of sleeve and clutch gear. In addition, the $I_{\max }$ has a significant increase when $F_{s} \geq 200 \mathrm{~N}$, and in this case, the impact would damage the splined teeth of the sleeve and the synchro ring terribly. When designing a gear-shifting control system for an AMT, it is advisable to avoid this region.

\section{Concluding remarks}

In the present paper, state-of-the-art techniques of hybrid system modeling and multibody dynamics have been applied to and adapted for the modeling and analysis of the engaging process of sleeve with clutch gear in automated mechanical transmissions. The engaging process is treated as a two-phase process-sleeve interacting with synchro ring and then with clutch gear. For the first phase, five cases are defined based on the relative rotational speed of sleeve and clutch gear. For the second phase, five cases are defined based on the relative position of sleeve and clutch gear. An impact model based on the Poisson coefficient of restitution is built to describe the impacts on the tooth chamfers of synchro ring and clutch gear. A hybrid automaton (HA) model is well defined and can cover the multiple interaction cases between the sleeve, synchro ring, clutch gear, etc. Bench tests are carried out to validate the high fidelity of the HA model. Numerical simulations are based on the validated HA model, and results show that the trajectories of the engaging-related parts have both continuous evolutions and discrete transitions, which can figure out the engaging duration and the impact impulse.

The characteristic analysis is focused on the effects of the relative position of sleeve and clutch gear and the shifting force on the performance of the engaging process, and the main results are: 
(1) The relative position of sleeve and clutch gear has a significant effect on the interaction way of sleeve and clutch gear. The engaging duration of the "obverse contacting + forward + reverse contacting" is longer than other cases. The "reverse contacting" case occupies the most $(60 \%)$ of the relative position.

(2) Increasing the shifting force can decrease the engaging duration but increase the maximum impact impulse. When the shifting force exceeds a threshold value, the generating mechanism of the maximum impact switches from the "sleeve contacts with clutch gear" to the "sleeve contacts with synchro ring," and at the same time, the value of the maximum impact impulse has a significant increase.

The analysis results in this paper can help engineers design a gear-shifting control system and improve the transmission configuration. The developed HA model is also effective for the gear-shifting control system verification and validation.

Acknowledgements This work was supported by the Ministry of Science and Technology of China under a "973" project (2011CB711202).

Open Access This article is distributed under the terms of the Creative Commons Attribution 4.0 International License (http://creativecommons.org/licenses/by/4.0/), which permits unrestricted use, distribution, and reproduction in any medium, provided you give appropriate credit to the original author(s) and the source, provide a link to the Creative Commons license, and indicate if changes were made.

\section{Appendix A: Configuration parameters of a mechanical transmission}

$m_{s l v} \quad$ Mass of sleeve

$m_{\text {syn }} \quad$ Mass of synchro ring

$m_{\text {slid }}$ Mass of sliding block

$J_{\text {out }} \quad$ Equivalent moment of inertia at transmission output shaft

$J_{s y n} \quad$ Moment of inertia of synchro ring

$J_{\text {in }} \quad$ Equivalent moment of inertia at clutch gear

$r_{g r} \quad$ Radius of clutch gear (Fig. 3)

$r_{\text {cone }}$ Radius of the cone of friction (Fig. 3)

$\alpha_{\text {cone }}$ Conical angle of synchro ring and clutch gear (Fig. 3)

$\alpha_{s l v} \quad$ Inner angle of sleeve (Fig. 3)

$\alpha_{g r} \quad$ Tooth chamfer angle of sleeve, synchro ring and clutch gear (Fig. 2)

$N \quad$ Tooth number of sleeve and clutch gear

$\zeta \quad$ Poisson coefficient of restitution between tooth chamfers

$\mu_{\text {slvgr }}$ Dynamic coefficient of friction between splined teeth of sleeve and clutch gear

$\mu_{\text {cone }}$ Dynamic coefficient of friction between cones of friction

$x_{1} \quad$ Axial position that sliding block contacts with the left end face of synchro ring

$x_{2} \quad$ Axial position that inner cone of synchro ring contacts with outer cone of clutch gear

$x_{3} \quad$ Axial position that sleeve teeth start to contact with tooth chamfers of synchro ring

$x_{4} \quad$ Axial position that sleeve teeth contact with tooth chamfers of synchro ring completely

$x_{5} \quad$ Axial position that sleeve engages with synchro ring

$x_{6} \quad$ Axial position that sleeve teeth start to contact with the tooth chamfers of clutch gear

$x_{7} \quad$ Axial position that the sleeve engages with the clutch gear

$x_{8} \quad$ Axial position that sleeve arrives at the self-locking position of desired clutch gear 


\section{Appendix B: Multibody dynamics}

B.1 Dynamics in $\ell_{1}$

The $v_{s l v}, \omega_{s l v}$, and $\omega_{g r}$ evolve according to the differential equations

$$
\left\{\begin{array}{l}
\dot{v}_{s l v}=\frac{F_{s}}{m_{s l v}+m_{s l i d}}, \\
\dot{\omega}_{s l v}=-\frac{T_{f v}}{J_{s y n}+J_{o u t}}, \\
\dot{\omega}_{g r}=-\frac{T_{f g}}{J_{\text {in }}} .
\end{array}\right.
$$

\section{B.2 Dynamics in $\ell_{2}$}

The $\omega_{s l v}$ and $\omega_{g r}$ evolve according to the same differential equations in (7). The $v_{s l v}$ evolves according to the differential equation

$$
\dot{v}_{s l v}=\frac{F_{s}}{m_{s l v}+m_{s l i d}+m_{s y n}} .
$$

\section{B.3 Dynamics in $\ell_{3}$}

As shown in Fig. 3, a contact force $F_{\text {slvsp }}$ is present between the sleeve and the spring plunger, and an axial force $F_{s y n}$ is present between the end faces of the sliding block and the synchro ring. We assume that the spring force $F_{f s}$ remains (almost) unchanged during the axial movement of sleeve, and then $F_{s y n}=\tan \left(\alpha_{s l v}\right) \cdot F_{f s}$ for the position holding of the spring plunger at axial direction [10]. With the axial force $F_{s y n}$, a dynamic frictional torque $T_{s y n}$ is present on the inner conical surface of the synchro ring to accelerate its rotation relative to the sleeve, where

$$
T_{\text {syn }}=\frac{r_{\text {cone }} \cdot \mu_{\text {cone }} \cdot F_{\text {syn }}}{\sin \left(\alpha_{\text {cone }}\right)} .
$$

Then, the $v_{s l v}, \omega_{s l v}, \omega_{s y n}$, and $\omega_{g r}$ evolve according to the differential equations

$$
\left\{\begin{array}{l}
\dot{v}_{s l v}=\frac{F_{s}}{m_{s l v}}-\frac{\tan \left(\alpha_{s l v}\right) \cdot F_{f s}}{m_{s l v}}, \\
\dot{\omega}_{s l v}=-\frac{T_{f v}}{J_{\text {out }}+J_{s y n}}, \\
\dot{\omega}_{s y n}=\frac{\tan \left(\alpha_{s l v}\right) \cdot r_{\text {cone }} \cdot \mu_{\text {cone }} \cdot F_{f s}}{\sin \left(\alpha_{\text {cone }}\right) \cdot J_{s y n}}, \\
\dot{\omega}_{g r}=-\frac{T_{f g}}{J_{\text {in }}}-\frac{\tan \left(\alpha_{s l v}\right) \cdot r_{\text {cone }} \cdot \mu_{\text {cone }} \cdot F_{f s}}{\sin \left(\alpha_{\text {cone }}\right) \cdot J_{\text {in }}} .
\end{array}\right.
$$

\section{B.4 Dynamics in $\ell_{4}$}

With reference to the $\ell_{3}$, we could obtain the dynamics in $\ell_{4}$. 


\section{B.5 Dynamics in $\ell_{5}$}

The $v_{s l v}$ and $\omega_{s l v}$ evolve according to the same differential equations in (10), and the $\omega_{s y n}$ evolves according to the differential equation

$$
\dot{\omega}_{s y n}=-\frac{T_{f g}}{J_{i n}+J_{s y n}} .
$$

B.6 Dynamics in $\ell_{6}$

The $v_{s l v}$ evolves according to the same differential equation in (10), and the $\omega_{g r}$ evolves according to the differential equation

$$
\dot{\omega}_{g r}=-\frac{T_{f g}+T_{f v}}{J_{\text {in }}+J_{\text {syn }}+J_{\text {out }}} .
$$

\section{B.7 Dynamics in $\ell_{7}$}

The $v_{s l v}$ and $\omega_{g r}$ evolve according to the same differential equations in (10), and the $\omega_{s y n}$ evolves according to the differential equation

$$
\dot{\omega}_{\text {syn }}=\frac{\tan \left(\alpha_{\text {slv }}\right) \cdot r_{\text {cone }} \cdot \mu_{\text {cone }} \cdot F_{f s}}{\sin \left(\alpha_{\text {cone }}\right) \cdot\left(J_{\text {syn }}+J_{\text {out }}\right)}-\frac{T_{f v}}{J_{\text {syn }}+J_{\text {out }}} .
$$

B.8 Dynamics in $\ell_{8}$

With reference to the $\ell_{7}$, we could obtain the dynamics in $\ell_{8}$.

B.9 Dynamics in $\ell_{9}$

The $\omega_{s l v}$ and the $\omega_{g r}$ evolve according to the differential equations

$$
\left\{\begin{array}{l}
\dot{\omega}_{s l v}=\frac{r_{\text {cone }} \cdot \mu_{\text {cone }} \cdot F_{s}}{\sin \left(\alpha_{\text {cone }}\right) \cdot\left(J_{\text {syn }}+J_{\text {out }}\right)}-\frac{T_{f v}}{J_{\text {syn }}+J_{\text {out }}}, \\
\dot{\omega}_{g r}=-\frac{r_{\text {cone }} \cdot \mu_{\text {cone }} \cdot F_{s}}{\sin \left(\alpha_{\text {cone }}\right) \cdot J_{\text {in }}}-\frac{T_{f g}}{J_{\text {in }}} .
\end{array}\right.
$$

B.10 Dynamics in $\ell_{10}$

With reference to the $\ell_{9}$, we could obtain the dynamics in $\ell_{10}$.

B.11 Dynamics in $\ell_{11}$

The $v_{s l v}, \omega_{s l v}$, and $\omega_{s y n}$ evolve according to the differential equations

$$
\left\{\begin{array}{l}
\dot{v}_{s l v}=\frac{F_{s}-\sin \left(\alpha_{g r}\right) \cdot N_{1}}{m_{s l v}}, \\
\dot{\omega}_{s l v}=\frac{\cos \left(\alpha_{g r}\right) \cdot r_{g r} \cdot N_{1}-T_{f v}}{J_{s y n}+J_{o u t}}, \\
\dot{\omega}_{s y n}=-\frac{T_{f g}+\cos \left(\alpha_{g r}\right) \cdot r_{g r} \cdot N_{1}}{J_{\text {in }}+J_{s y n}},
\end{array}\right.
$$


where $N_{1}$ represents the contact force at the lower tooth chamfer of synchro ring. With a constraint equation $\left(\dot{\omega}_{s l v}-\dot{\omega}_{s y n}\right) \cdot r_{g r}=\dot{v}_{s l v} \cdot \tan \left(\alpha_{g r}\right)$, the $N_{1}$ is obtained as

$$
N_{1}=a_{1} \cdot T_{f g}+a_{2} \cdot T_{f v}+a_{3} \cdot F_{s},
$$

where

$$
\begin{aligned}
& a_{1}=-\frac{r_{g r}}{\left(J_{\text {in }}+J_{\text {syn }}\right) \cdot K}, \\
& a_{2}=\frac{r_{g r}}{\left(J_{\text {syn }}+J_{\text {out }}\right) \cdot K}, \\
& a_{3}=\frac{\tan \left(\alpha_{g r}\right)}{m_{\text {slv }} \cdot K},
\end{aligned}
$$

where

$$
K=r_{g r}^{2} \cdot\left[\frac{\cos \left(\alpha_{g r}\right)}{J_{\text {in }}+J_{\text {syn }}}+\frac{\cos \left(\alpha_{g r}\right)}{J_{\text {syn }}+J_{\text {out }}}\right]+\frac{\tan \left(\alpha_{g r}\right) \cdot \sin \left(\alpha_{g r}\right)}{m_{s l v}} .
$$

B.12 Dynamics in $\ell_{12}$

With reference to the $\ell_{11}$, we could obtain the dynamics in $\ell_{12}$.

B.13 Dynamics in $\ell_{13}$

The $v_{s l v}, \omega_{s l v}$, and $\omega_{g r}$ evolve according to the differential equations

$$
\left\{\begin{array}{l}
\dot{v}_{s l v}=\frac{F_{s}}{m_{s l v}}, \\
\dot{\omega}_{s l v}=-\frac{T_{f v}}{J_{s y n}+J_{o u t}}, \\
\dot{\omega}_{g r}=-\frac{T_{f g}}{J_{\text {in }}} .
\end{array}\right.
$$

B.14 Dynamics in $\ell_{14}$

The $v_{s l v}$ and $\omega_{g r}$ evolve according to the differential equations

$$
\left\{\begin{array}{l}
\dot{v}_{s l v}=\frac{F_{s}}{m_{s l v}}+\frac{\mu_{s l v g r} \cdot T_{f g} \cdot\left(J_{s y n}+J_{\text {out }}\right)-\mu_{\text {slvgr }} \cdot T_{f v} \cdot J_{\text {in }}}{m_{s l v} \cdot r_{g r} \cdot\left(J_{\text {in }}+J_{\text {syn }}+J_{\text {out }}\right)}, \\
\dot{\omega}_{g r}=-\frac{T_{f g}+T_{f v}}{J_{\text {in }}+J_{\text {syn }}+J_{\text {out }}} .
\end{array}\right.
$$

B.15 Dynamics in $\ell_{15}$

The $\omega_{g r}$ evolves according to the same differential equation in (18). 


\section{References}

1. Chen, H., Mitra, S.: Synthesis and verification of motor-transmission shift controller for electric vehicles. In: 2014 ACM/IEEE International Conference on Cyber-Physical Systems (ICCPS), pp. 25-35. IEEE Press, New York (2014)

2. Gianluca Lucente, C.R., Montanari, M.: Modelling of an automated manual transmission system. Mechatronics 17, 73-91 (2007)

3. Hoshino, H.: Analysis on synchronization mechanism of transmission. Technical report, SAE Technical Paper (1999)

4. Kim, J., Sung, D., Seok, C., Kim, H., Song, H., Lim, C., Kim, J.: Development of shift feeling simulator for a manual transmission. Technical report, SAE Technical Paper (2002)

5. Liu, Y.-C., Tseng, C.-H.: Simulation and analysis of synchronisation and engagement on manual transmission gearbox. Int. J. Veh. Des. 43(1), 200-220 (2007)

6. Lovas, L., Play, D., Márialigeti, J., Rigal, J.-F.: Mechanical behaviour simulation for synchromesh mechanism improvements. Proc. Inst. Mech. Eng., Part D, J. Automob. Eng. Mech. 220(7), 919-945 (2006)

7. Lovas, L., Play, D., Márialigeti, J., Rigal, J.-F.: Modelling of gear changing behaviour. Period. Polytech., Transp. Eng. 34(1-2), 35-58 (2006)

8. Marco, J., Ball, R., Jones, R., Lillie, K.: A systems modelling and simulation approach to gear shift effort analysis. Int. J. Veh. Des. 25(4), 317-338 (2001)

9. Naunheimer, H., Bertsche, B., Ryborz, J., Novak, W.: Automotive Transmissions: Fundamentals, Selection, Design and Application. Springer, Berlin (2010)

10. Razzacki, S.T.: Synchronizer design: a mathematical and dimensional treatise. Technical report, SAE Technical Paper (2004)

11. Rosen, I., Kruk, S., Eker, P., Mellgren, H.: Synchromesh mechanisms: experience of heavy truck gearboxes. Proc. Inst. Mech. Eng. 184(9), 438-476 (1969)

12. Seifried, R., Schiehlen, W., Eberhard, P.: Numerical and experimental evaluation of the coefficient of restitution for repeated impacts. Int. J. Impact Eng. 32(1), 508-524 (2005)

13. Shusen, L., Siqin, C., Bo, L.: Gearshift control for amt based on a 2-dof electromagnetic actuator. Trans. Chin. Soc. Agric. Mach. 4, 003 (2014)

14. Socin, R.J., Walters, L.K.: Manual transmission synchronizers. Technical report, SAE Technical Paper (1968)

15. Tseng, C.-Y., Yu, C.-H.: Advanced shifting control of synchronizer mechanisms for clutchless automatic manual transmission in an electric vehicle. Mech. Mach. Theory 84, 37-56 (2015) 